\title{
Analyses of Code Division Multiple Access (CDMA) Schemes for Global Mobile Satellite Communications (GMSC)
}

\author{
D.S. Ilcev \\ Durban University of Technology, Durban, South Africa
}

\begin{abstract}
This paper describes in particular Code Division Multiple Access (CDMA) applicable in Global Mobile Satellite Communications (GMSC). In satellite communication systems, as a rule, especially in GMSC networks many users are active at the same time. The problem of simultaneous communications between many single or multipoint mobile satellite users, however, can be solved by using Multiple Access Technique (MAT) system. Since the resources of the systems such as the transmitting power and the bandwidth are limited, it is advisable to use the channels with complete charge and to create a different MAT to the channel. This generates a problem of summation and separation of signals in the transmission and reception parts, respectively. Deciding this problem consists in the development of orthogonal channels of transmission in order to divide signals from various users unambiguously on the reception part.
\end{abstract}

\section{INTRODUCTION}

The Code Division Multiple Access (CDMA) technology has been known for a long time. Namely, in the Soviet Union (USSR), the first work dedicated to this muliplexing technique and solutions for wireless communication applications was published in 1935 by Russian scientist Dmitry Ageev. During experiments conducted at that time, it was shown that by applying linear methods, three types of signal separation appear: frequency, time, and compensation. Accordingly, for the first time, the CDMA technology was used practically in 1957, when a young Rusian military radio engineer, Leonid Kupriyanovich, made in Moscow his first experimental model of a portable automatic mobile phone system, called the LK-1, with a base station. In effect, the LK-1 mobile phone, as a precursor to today's mobile phones, had a weight of approximatelly $3 \mathrm{~kg}$, the range of its operation coverage was up to about $20-30 \mathrm{~km}$, and between 20 and 30 hours of battery life. The base station that serves mobile phones, as described by the author, could communicate simultaneously with several mobile users. However, in 1958, radio engineer Kuprijanovic made a new experiment with a "pocket" model of a mobile phone, which weighed only $0.5 \mathrm{~kg}$. In order to serve a larger number of customers, Kuprijanovic proposed a device he called a "correlator". Using the achievements of coding multiple acces in the development of mobile phones, in 1958, the intensive research and development have also begun in the USSR vehicular mobile phones known as "Altai", for national use in road vehicles and cars, based on the Soviet standard MRT-1327. This phone system weighed $11 \mathrm{~kg}(24 \mathrm{lb})$, which initially was placed in the trunk of the vehicles of high-ranking officials and used a standard handset in the passenger compartment. The main developers of the Altai system were Voronezh Science Research Institute of Communications (VNIIS) and State Specialized Project Institute (GSPI). In 1963 this 
service started in Moscow, and in 1970 Altai service was used in 30 USSR cities.

The CDMA technique is a method of channel access used by various radio communication technologies, therefore, the CDMA scheme is an example of multiple access, where several transmitters can simultaneously send information over a single communication channel. This allows several users to share the frequency band (see bandwidth). To enable this without unnecessary interference between users, CDMA uses spread spectrum technology and a special coding scheme (where each transmitter is assigned a code). This technique allows several users to share the frequency band by spoecific separation. To enable this without unnecessary interference between users, CDMA uses spread spectrum technology and a special coding scheme, where each transmitter is assigned a code. The CDMA sceme is used as an access method in many mobile phone standards. The IS-95, also called "cdmaOne", and its 3G evolutionary CDMA2000 are often referred to as "CDMA", but UMTS, the 3G standard used by mobile operators, also uses "broadband CDMA" or V-CDMA, as well as TDCDMA. and TD-SCDMA, as its radio technologies. The succesor for 4G CDMA2000 was Ultra Mobile Broadband (UMB), however, in November 2008, The US little know company named Qualcomm Incorporation announced that it was completing technology development, favoring Long-Term Evolution (LTE).

Therefore, the CDMA technique is based around a special form of transmission known as Direct Sequence Spread Spectrum (DSSS) scheme, which history can be directly linked back to the 1940s when this form of transmission was first envisaged. As electronics technology improved, it started to be used for covert military witeless communication in view of the facts that the transmissions look like noise, it is difficult to decipher without the knowledge of the right codes, and furthermore it is difficult to jam. With the revolution in cellular telecommunications that occurred in the 1980s a Qualcomm Incorporation working on DSSS transmissions started to look at this as the basis for a CDMA as cellular telecommunications multiple access scheme.

The concept of CDMA had to proved in the field and accordingly the Qualcomm company was joined by US network operators Nynex and Ameritech to develop the first experimental CDMA system. Later the mutual team was expanded as Motorola and AT\&T (now Lucent) joined to bring their resources to speed development. As a result this it was possible to start writing a specification for CDMA project in 1990. With the support of the Cellular Telecommunications Industry Association (CTIA) and the Telecommunications Industry Association (TIA) a standards group was set up. Therefore, an oficially the CDMA technique was introduced in 1995 and soon become the fastest growing wireless technology.

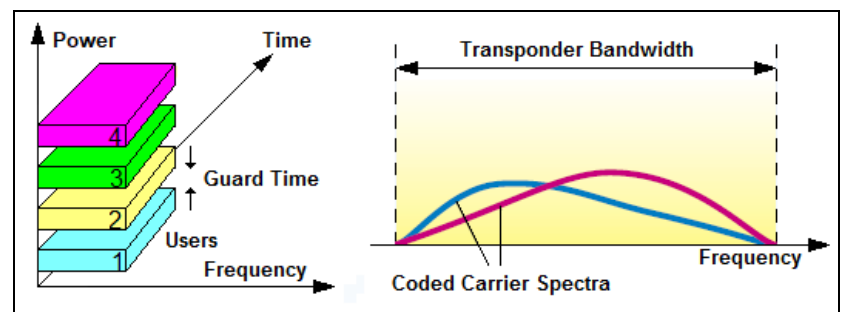

Figure 1. Code Division Multiple Access Technique and CDMA Carrier Spectra

Afterwards, this standard group of CTIA and TIA then published the standard for the first CDMA system in the form of IS-95, resulting in the formal publication of IS-95-A in 1995. The first CDMA system was launched in September 1995 by Hutchison Telephone Co. Ltd. in Hong Kong and SK Telecom in Korea soon followed along with networks in the USA. This was only one cellular telecommunications system, although it was the first. Its development lead on to the DMA2000 series of standards. The use of CDMA did not stop with CDMA2000 as it became necessary to evolve the cellular Global System for Mobile (GSM) standard so that it could carry data and provide significant improvements in terms of spectrum use efficiency. Accordingly CDMA scheme, in the form of Wideband CDMA (WCDMA) was adopted for this standard.

The US Loral Space \& Communications company, with Qualcomm Incorporation in 1998 developed the concept of Globalstar GMSC system that uses CDMA and Frequency Division Multiple Access (FDMA) methods for mobile satellite communications. The Globalstar CDMA technique is a modified version of the IS-95, which was originally developed by Qualcomm for Cellular 3G communication services world wide. Pioneered by Qualcomm the CDMA signal provides excellent data and voice capacity through the Globalstar mobile satellite phone network of 48 satellites. Globalstar chose CDMA system for use in it satellite communication network when Globalstar launched service in 2000. The CDMA technique converts speech signal into digital format and then transmits it from the Globalstar Satellite phone up to the satellite systems and down to the ground station. Every call over the satellite network has its own unique code which distinguishes it from the other calls sharing the airwaves at the same time. The CDMA signal is without interference, cross talk or static.

\section{PRINCIPLES OF CDMA SCHEME}

In the CDMA system all fixed and mobile users occupy the total transponder bandwidth all the time. The users can be separated because each channel is multiplied by a unique spreading code. The composite signal is then modulated onto a carrier. The information is recovered by multiplying the demodulated signal with the same spreading code. For instance, the receiving Mobile Earth Station (MES) terminal is accordingly able to recover the transmitted message by a specific user from schore. No frequency or time coordination is needed before 
accessing the transponder, so new users can easily be included and the CDMA signals are resistant to interfering signals from other users. This property can be utilised in systems with Very Small Aperture Terminals (VSAT) stations for fixed and mobile applications, where interference may be received from adjacent satellites. The main disadvantages are cost and complexity of the receivers. The simplest form of satellite VSAT networks is the point-to-point or point-to-multipoint configuration conecting groind consumers with MES terminals via Ground Earth Stations (GES) and Geostationary Earth Orbit (GEO) and Non-GEO satellites. Usually, for GMSC networks can be used Medium Earth Orbit (MEO) or Little Earth Orbit (LEO) satellite constellations.

The CDMA solution is based on the use of the modulation technique also known as Spread Spectrum Multiple Access (SSMA), which means that it spreads the information contained in a particular signal of interest over a much greater bandwidth than the original signal. In this MA scheme, the resources of both frequency bandwidth and time are shared by all users employing orthogonal codes, which scheme is shown in Figure 1. (Left). The CDMA scheme is achieved by a PN (Pseudo-Noise) sequence generated by irreducible polynomials, which is the most popular CDMA method. In this way, an SSMA method using low-rate error-correcting codes, including orthogonal codes with Hadamard or waveform transformation has also been proposed.

Therefore, the SDMA system is a scheme where all concerned Earth stations can use the same frequency at the same time within a separate space available for each link. The satellite transponder is a resource that can be characterized by its available power and bandwidth, which configuration of CDMA carriers spectra is illustrated in Figure 1 (Right). The efficient use of this common resource is a very important problem in satellite communication. The channels are designed to the users either fixed (pre-assigned) or on-demand (demand assignments). Thus, the MAT configuration permits more than two Earth stations to use the same satellite network for interchanging information. Several transponders in the satellite payload share the frequency bands in use and each transponder will act independently of the others to filter out its own allocated frequency and further process that signal for transmission. This feature allows any MES terminal located in the corresponding coverage area to receive carriers originating from several MES terminals and vice versa and carriers transmitted by one MES can be received by any GES terminal. This enables a transmitting Earth station to group several signals into a single, multi-destination carrier. Access to a transponder may be limited to a single carrier or many carriers may exist simultaneously. The baseband information to be transmitted is impressed on the carrier by the single process of multi-channel modulation.

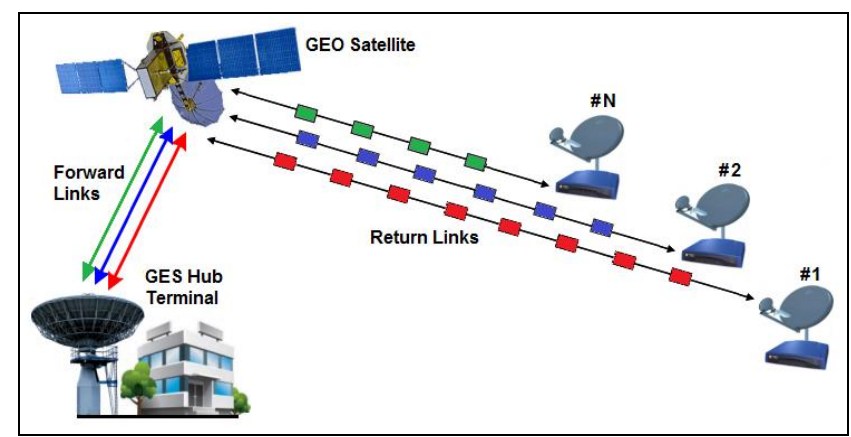

Figure 2. Satellite CDMA Network Architecture

Concerning the specific encoding process, each user is actually assigned a signature sequence, with its own characteristic code, chosen from a set of codes assigned individually to the various users of the system. This code is mixed, as a supplementary modulation, with the useful information signal. On the reception side, from all the signals that are received, a given mobile user is able to select and recognize, by its own code, the signal, which is intended for it, and then to extract useful information. The other received signal can be intended for other users but they can also originate from unwanted emissions, which gives CDMA a certain anti-jamming capability. For this operation, where it is necessary to identify one CDMA transmission signal among several others sharing the same band at the same time, correlation techniques are generally employed. From a commercial and military perspective, this MAT is still new and has significant advantages. Interference from adjacent satellite systems including jammers is better solved than with other systems. This scheme is simple to operate as it requires no synchronization of the transmitter and is more suited for a military system. Small mobile antennas can be useful in these applications, without the interference caused by wide antenna bandwidths.

Using multibeam satellites, frequency reuse with CDMA is very effective and allows good flexibility in the management of traffic, and the orbit/spectrum resources. The Power Flux Density (PFD) of the CDMA signal received in the service area is automatically limited, with no need for any other dispersal processes. It also provides a low probability of intercept of the users and some kind of privacy, due to individual characteristic codes. The main disadvantage of CDMA by satellite is that the bandwidth required for the space segment of the spread carrier is very large, compared to that of a single unspread carrier, so the throughput is somewhat lower than with other systems.

Therefore, in the CDMA scheme, the signals from various users operate simultaneously, at the same nominal frequency but are spread in the given allocated bandwidth by a special encoding process. Depending on the multiplexing techniques employed the bandwidth may extend to the entire capacity of the transponder but is often restricted to its own part, so CDMA can possibly be combined in the hybrid scheme with FDMA and/or TDMA. The SSMA technique can be classified into two methods: Direct Sequence (DS) and Frequency Hopping (FH). A combined system of DC and FH is called a hybrid 
CDMA system and the processing gain can be improved without increases in chip rate.

At present, the CDMA system advantages are practically effective in new satellite systems, such as Globalstar, also developed by Qualcomm, which is devoted to MSS handheld terminals, and Skybridge, involved in FSS. This type of MA is therefore attractive for handheld and portable MSS equipment with a wide antenna pattern. Antennas with large beam widths can otherwise create or be subject to interference with adjacent satellites. In any case, this MA technique is very attractive for commercial, military, and even TT\&C communications because some Russian satellites use CDMA for command and telemetry purposes. The Synchronous-CDMA (SCDMA) scheme proves efficiently to eliminate interference arising from other users sharing the same carrier and the same spot beam. Interference from other spot beams that overlap the coverage of the intended spot is still considerable. This process to ensure orthogonality between all links requires signaling to adjust transmission in time and frequency domains for every user independently.

\section{CODE DIVISION MULTIPLE ACCESS (CDMA) NETWORK CONCEPT}

As outlined earlier, the CDMA technique is the earliest implemented in the wireless systems and still one of the most commonly employed forms of multiple access techniques for communications via satellite systems. In the case of CDMA different Earth stations are able to access the total available bandwidth of satellite transponder by virtue of their different carrier frequencies and time, thus avoiding interference among multiple signals. Therefore, as a third fundamental MAT system, the CDMA is a combination of both frequency and time separation. In fact, it is the most complex technique to implement, requiring several levels of synchronization at both the transmission and reception levels. The CDMA scheme is practical for digital formatted data only and offers the highest power and spectral efficiency operation of the three fundamental techniques.

The functional display of the CDMA process is similar in presentation to those for two fundamental FDMA and TDMA systems. Thus, each uplink station is assigned a time slot and a frequency band in a coded sequence to transmit its station packets. A forward uplink link is the transmission direction from a fixed location, such as a GES terminal or a base station in wirelless systems to a fixed or mobile (MES) VSAT stations via LEO/MEO/GEO satellites, which scenario for CDMA system is shown in Figure 2.

If the link includes a communications relay satellite, the forward link will consist of both an uplink, GES terminal or a base station to satellites, and a downlink, from satellites to MES or cellular terminals. A return uplink is transmision direction from a fixedor mobile location, such as fixed or mobile (MES) VSAT of the GES terminal or a base station in wireless systems. If the link includes a communications relay satellite, the return link will consist of both an uplink, from fixed or mobile (MES) VSAT stations to LEO/MEO/GEO satellites, and a downlink, from satellites to the fixed or mobile (MES) or cellular terminals.

The forward downlink receive station must know the code of frequency and time locations in order to detect the complete data sequence. The receive station (GES terminal) with knowledge of the code can recoup the signal from the noise-like signal that appears to a receiver that does not know the code. As stated, the CDMA scheme is often referred to as the SSMA technique because of the signal spreading characteristics of the process, which is achieved by a PN (Pseudo-Noise) sequence generated by irreducible polynomials, which is the most popular CDMA method. In this way, an SSMA method using low-rate error-correcting codes, including orthogonal codes with Hadamard or waveform transformation has also been proposed.

\subsection{Direct Sequence (DS) CDMA}

In telecommunications, direct-sequence spread spectrum is a spread-spectrum modulation technique primarily used to reduce overall signal interference. The direct-sequence modulation makes the transmitted signal wider in bandwidth than the information bandwidth. The data is divided and simultaneously transmitted on as many frequencies as possible within a particular frequency band (the channel). It adds redundant bits of data known as chips to the data to represent binary 0 s or $1 \mathrm{~s}$. The ratio of chips to data is known as the spreading ratio: the higher the ratio, the more immune to interference the signal is, because if part of the transmission is corrupted, the data can still be recovered from the remaining part of the chipping code. This dominant DS-CDMA technique is also called Pseudo-Noise (PN) modulation, where the modulated signal is multiplied by a PN code generator, which generates a pseudo-random binary sequence of length $(\mathrm{N})$ at a chip rate $(\mathrm{Rc})$, much larger than the information bit rate $(\mathrm{Rb})$. The chip rate sequence is introduced by the following relation:

$\mathrm{Rc}=\mathrm{N} \cdot \mathrm{Rb}$

This sequence is combined with the information signal cut into small chip rates (Rc), thus, speeding the combined signal in a much larger bandwidth $(\mathrm{W} \sim \mathrm{Rc})$. Namely, the resulting signal has a wider frequency bandwidth than the original modulated signal. In such a way, the transmitting signal can be expressed in the following way:

$s(t)=m(t) p(t) \cos (2 \pi f c t)=m(t) p(t) \cos \omega c t$

where values $m(t)=$ binary message to be transmitted and $\mathrm{p}(\mathrm{t})=$ spreading NP binary sequence. Consequently, at the GES receiver, the satellite signal is coherently demodulated by multiplying the received signal by a replica of the carrier. Neglecting thermal noise, the receiving signal at the input of the detector of Low-Pass Filter (LPF) is given by the following relation: 
$\mathrm{r}(\mathrm{t})=\mathrm{m}(\mathrm{t}) \mathrm{p}(\mathrm{t}) \cos \omega \mathrm{ct}(2 \cos \omega \mathrm{ct})=\mathrm{m}(\mathrm{t}) \mathrm{p}(\mathrm{t})+\mathrm{m}(\mathrm{t}) \mathrm{p}(\mathrm{t})$ $\cos 2 \omega c t$

The detector LLF eliminates the HF components and retains only the $\mathrm{LW}$ components, such as $\mathrm{u}(\mathrm{t})=$ $\mathrm{m}(\mathrm{t}) \mathrm{p}(\mathrm{t})$. This component is then multiplied by the local code $[p(t)]$ in phase with the received code, where the product $p(t) 2=1$. At the output of the multiplier this gives:

$$
x(t)=m(t) p(t) p(t)=m(t) p(t) 2=m(t) \quad[V]
$$

The signal is then integrated over one-bit period to filter the noise. The transmitted message is recovered at the integrator output, so in fact, only the same PN code can achieve the despreading of the received signal bandwidth.

In this process, the interference or jamming spectrum is spread by the PN codes, while other user's signals, spared by different PN codes, are not despread. Interference or jamming power density in the bandwidth of the received signal decreases from their original power.

Otherwise, the most widely accepted measure of interference rejection is the processing gain $(\mathrm{Gp})$, which is given by the ratio $\mathrm{Rc} / \mathrm{Rb}$ and value of $\mathrm{Gp}=$ $20-60 \mathrm{~dB}$. The input and output signal-to-noise ratios are related as follows:

\section{$(\mathrm{S} / \mathrm{N})$ Output $=\mathrm{Gp}(\mathrm{S} / \mathrm{N})$ Input}

In the forward link, the LES (Hub Station) transmits the spread-spectrum signals, which are spread with synchronized PN sequence to different MSC users. Since orthogonal codes can be used, the mutual interference in the network is negligible and the channel capacity is close to that of FDMA. Conversely, in the return link, the signals transmitted from different MES users are not synchronized and they are not orthogonal. The first case is referred to as synchronous and the second case as asynchronous SSMA.

However, the nonorthogonality causes interference due to the transmission of other MES in the satellite network and as the number of simultaneously accessing users increases, the communication quality gradually degrades in a process called Graceful Degradation.

\subsection{Frequency Hopping (FH) CDMA}

The FH-CDMA system works similarly to the DS system since a correlation process of de-hopping is also performed at the receiver. The difference is that here the pseudo-random sequence is used to control a frequency synthesizer, which results in the transmission of each information bit rate in the form of $(\mathrm{N})$ multiple pulses at different frequencies in an extended bandwidth. The transmitted and received signals have the following forms:

$\mathrm{s}(\mathrm{t})=\mathrm{m}(\mathrm{t}) \cos \omega \mathrm{c}(\mathrm{t}) \mathrm{t}$ $r(t)=m(t) \cos \omega c(t) t \cdot 2 \cos \omega c(t) t=m(t)+m(t) \cos$

$2 \omega c(t) t$

Thus, at the receiver, the carrier is multiplied by an unmodulated carrier generated under the same conditions as at the transmitter. The second term in the receiver is eliminated by the LPF of the demodulator. The relation of processing gain for $\mathrm{FH}$ is:

$\mathrm{Gp}=\mathrm{W} / \Delta \mathrm{f}$

where $\mathrm{W}=$ frequency bandwidth and $\Delta \mathrm{f}=$ bandwidth of the original modulated signal. At this point, coherent demodulation is difficult to implement in $\mathrm{FH}$ receivers because it is a problem to maintain phase relation between the frequency steps. Due to the relatively slow operation of the frequency synthesizer, DS schemes permit higher code rates than $\mathrm{FH}$ radio systems.

\section{CONCLUSION}

The performances and capacities of MSC for CDMA, FDMA, and TDMA/FDMA have been analyzed many years ago for an L/C-band network with global coverage. For the particular MSS under discussion and for the particular antenna configurations, both CDMA and FDMA offer similar performance, FDMA yielding slightly higher channel capacities at the design point and CDMA being slightly better at higher EIRP levels. As the MSS grows and the antenna beam size decreases, CDMA appears to be a very efficient system, because it is not limited by Lband bandwidth constraints. However, CDMA is wasteful in feeder link bandwidth, and the choice of a multiple access system must take all parameters into consideration, such as oscillator stability, interference rejection, system complexity, etc. as well as system cost before deciding on a particular multiple access systems.

The communication satellites for MSC provide multiple-beam antennas and employ frequency reuse of the allocated L-band frequency spectrum. It appears that despite the fact that FDMA and FDMA/TDMA are orthogonal systems, they nevertheless suffer from bandwidth limitations and sensitivity to interbeam interference in L-band.

The CDMA scheme is better at absorbing Doppler and multipath effects, and it permits higher rate coding, but it suffers from self-jamming and from bandwidth constraints in the feeder link. In general, all three multiple access systems show similar performance. However, at the chosen design point for aggregate EIRP, a number of beams, and allocated bandwidth, FDMA provides still the highest system channel capacity.

The narrowness of the frequency spectrum allocated to MSC means that it has to be explored to the full. Methods available for effective spectrum utilization include efficient signal design and subdivision of the total coverage area into narrow illumination zones. Modern satellites for MSC have 
also onboard processors, which connect an uplink band to a downlink beam. Processors use A/D conversion and digital filtering. The A/D converters quantize the signal and produce quantization noise.

Recently is developed SDMA as an advanced solution where all concerned MES terminals can share the same frequency at the same time within a separate space available for each link. On the other hand, the RDMA scheme is suitable for a large number of users in MSC, where all MES terminals share asynchronously the same transponder by randomly transmitting short burst or packet divisions. In addition is developed several mobile Aloha methods, which successfully increase the system throughout.

\section{REFERENCES}

[1] Gutteberg O., (1999), "Satellite Communications", Telektronikk, Telenor Group, Fornebu, Norway, 88.

[2] Popoola S.I., (2016), "Mobile \& Personal Communications", Department of Electrical and Information Engineering, Covenant University, Ota, Nigeria, 34.

[3] Richharia M., (2020), "Satellite Communication Systems", Springer, Boston, 484.

[4] Ilcev, (2017), "Global Mobile Satellite Communications for Maritime, Land and Aeronautical Applications Volume2", Springer, Boston, US, 686.

[5] Ivanov A., at all, (2019) Physical Layer Representation in LEO Satellite with a Hybrid Multi-Beamforming,
Skolkovo Institute of Science and Technology, Moscow, 7.

[6] Ilcev D. S. (2011), “Global Mobile CNS”, Manual, CNS Systems, Durban, South Africa, 285.

[7] Maini A.K. \& Agrawal V., (2007), "Satellite Technology - Principles and Applications", John Wiley, Chichester, 696.

[8] Garvey G., (2013), "Multiple Access Techniques", Atlanta RF, LLC, Roswell, GA, 53.

[9] Xu X., at all, (2018), "Combination of Asymmetric PCMA and Common Multiple Access Technology", Army Engineering University of PLA, Nanjing, China, 9.

[10] Pratt S.R. at all, (1999), "Operational and Performance Overview of the Iridium Low Earth Orbit Satellite System", IEEE Communications Surveys, New York, 9.

[11] Robert G. Winch R.G., (1993), "Telecommunication Transmission Systems", McGraw-Hill, New York, 633.

[12] Popoola S.I., at all, (2016), "Multiple Access Techniques: Design Issues in FDMA/TDMA", Manchester Metropolitan University, Manchester, UK, 34.

[13] Gigard R, (2020), "Satellite Network Design", Intelsat, McLean, VA, 53.

[14] Gagliardi R.M., (1991), "Satellite Communications", Van Nostrand Reinhold, New York, 592.

[15] Ohmory S., Wakana. H \& Kawase S. (1998), “Mobile Satellite Communications", Artech House, Boston, 466.

[16] Telecomunicazioni, (2008), "TDMA, FDMA, and CDMA" University of Rome La Sapienza, Rome, Italy, 42.

[17] Kim H.W., at all, (2012), Applicability of Orthogonal Frequency Division Multiple Access in Satellite Communication", ETRI, Daejon, South Korea, 6.

[18] Sheriff R.E. \& Hu Y.F., (2001), "Mobile satellite communication networks", John Wiley, Chichester, 381. 